\title{
Static Analysis of Coupled Micro - beams at both Ends of Electromechanical Coupling
}

\author{
Ning Sun ${ }^{1, a}$, Dawei Chen ${ }^{2, b}$ and Daolin Jiang ${ }^{2}$ \\ ${ }^{1}$ School of Mechanical and Electrical Engineering, Guilin University of Technology, Gulin 541004, \\ China \\ ${ }^{2}$ School of Mechanical and Electrical Engineering, Guilin University of Electronic Technology, Gulin \\ 541004, China \\ asunning@guet.edu.cn, b18593234802@163.com
}

Keywords: MEMS, Fixed beam at both ends, The deflection, Thickness.

\begin{abstract}
In the micro electro-mechanical system, the electrostatic coupling of the micro-beam at both ends is often used as the driving structure, so it is very important to study the static analysis of the micro-beam structure. The relationship between the geometric structure parameters and the maximum deflection is analyzed by using the Trans coupling unit of Trans 126 in ANASYS, and the relationship between the flexural rigidity and the thickness of the micro-beads in the micro electromechanical structure is further revealed.
\end{abstract}

\section{Introduction}

Micro Electro- Mechanical System ${ }^{[1]}$ (MEMS) is a micro-system integrating micro-sensors, micro-actuators, signal processing and control circuits. It is an organic integration of micromechanical and microelectronics. In the microstructure, the influence of its structural parameters on its performance also attracts a lot of attention. MEMS is a device or system that realizes the unique function through the conversion of energy, electricity and magnetism, and involves the coupling of various physical fields. So the micro-electromechanical system is a very complex system of multienergy domain coupling. Most MEMS devices are geometrically complexed There is a non-linear coupling situation, if the design is not properly, in a number of physical field of the combined effect of the case, the system is easy to fail. Therefore, it is necessary to study the response and effect of MEMS devices or systems under different energy domains, and correctly simulate and predict the behavior characteristics of the system.

Sun ${ }^{[2]}$ et al. Analyzed the film strain in the films by finite element analysis. The results of the analysis did not show the effect of the material parameters on the microsystems due to the calculated thickness. Eckstein and Engel ${ }^{[3]}$ The thickness of the micro-beam is reduced to a size comparable to the grain size when the micro-beam thickness is greater than 3 to 5 grains, and the bending force increases with the increase of the plate thickness. When the thickness of the micro beam to and grain size is, with the decrease of the thickness of sheet bending force and increased slightly. In this paper, ANSYS is used to calculate the thickness and deflection of the structural parameters under the condition of electromechanical coupling. The influence model of the thickness of different microstructures on the static driving performance is further revealed.

\section{Modeling Analysis of Fixed Beam at Two Ends}

\subsection{Simplified model of micro-beam structure driven by voltage}

The simplified model of the fixed beam at both ends is shown in figure 1, and the DC voltage is applied above the fixed beam at both ends. 


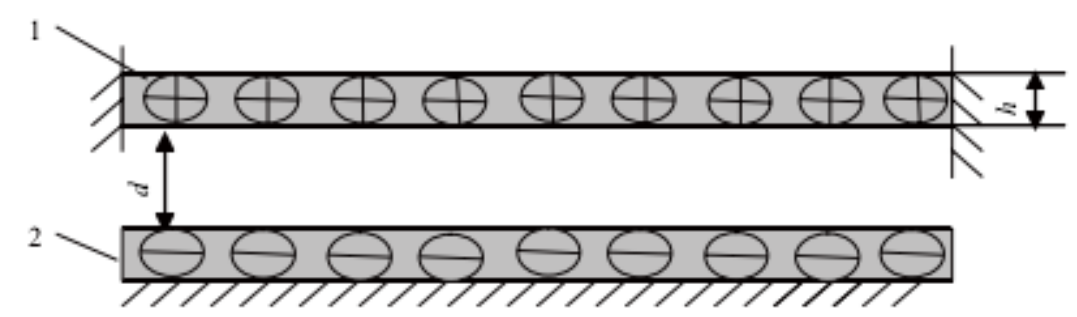

1. Clamped beam; 2. The basal

Fig. 1 Ends clamped beam section diagram

When loading the voltage between the upper and lower electrodes of the micro-beam at both ends, the upper he positive and negative charges are attracted to each other to produce the coulomb force, Of the static power, the Cullen force:

$$
F=\frac{q_{1} q_{2}}{4 \pi \varepsilon d_{0}^{2}}
$$

Annotation: $q_{1}$ and $q_{2}$ is Charge the battery;

$\varepsilon$ is Dielectric constant;

$d_{0}$ is Charge spacing.

It can be seen from the equation (1) that it is necessary to know the spacing between the two charges to calculate the Coulomb force to calculate the deflection of the micro-beam under the electrostatic force. In the microstructure, the charge distribution changes with the relative position and the shape of the structure. Therefore, it is very difficult to accurately determine the actual distribution of the charge on the structure. So the Trans126 order reduction unit in ANSYS is used to modeling simulation analysis.

\subsection{Size setting of micro-beam structure model}

The relevant structural parameters are as follows:

The vacuum dielectric constant as follows: $\mathcal{E}_{0}=8.854 e-6 \mathrm{pF} / \mathrm{um}$;

Modulus of elasticity is: $E=160 \mu N / \mu m^{2}$;

Poisson's ratio is: $v=0.22$;

The length of the beam is: $l=300 \mu \mathrm{m}$;

The width of the beam is: $b=30 \mu m$;

Initial spacing plate as follows: $d=3 \mu \mathrm{m}$;

The thickness of the beam h separately $3 \mu \mathrm{m}, 5 \mu \mathrm{m}, 7 \mu \mathrm{m}, 10 \mu \mathrm{m}, 15 \mu \mathrm{m}$.

\subsection{Analysis of micro-beam modeling}

The two ends of the fixed beam micro-beam based on the direct coupling method-TRANS 126 unit simulation analysis process:

1) Define the job name, define the analysis of the title - analysis of the static characteristics of the micro-beam;

2) define the type of unit, select the physical environment compatible with the type of unit SOLID122;

3) define the material properties, ex, nuxy, dens to define the elastic modulus of the micro-beam, Poisson's ratio, density; create a solid model;

4) meshing the solid model;

5) loading, the first section of the micro-beam to set the constraints; application EMTGEN command in a mobile structure of the surface (micro-beam surface) and a plane (lower electrode surface) between a series of TRANS126 unit on the electrostatic - Full coupling simulation

6) The electrostatic field solver was used to solve the finite element method, and the micro-beam was analyzed statistically. Through the ANSYS simulation of these five models to get a deformation map (shown in Figure 2). 


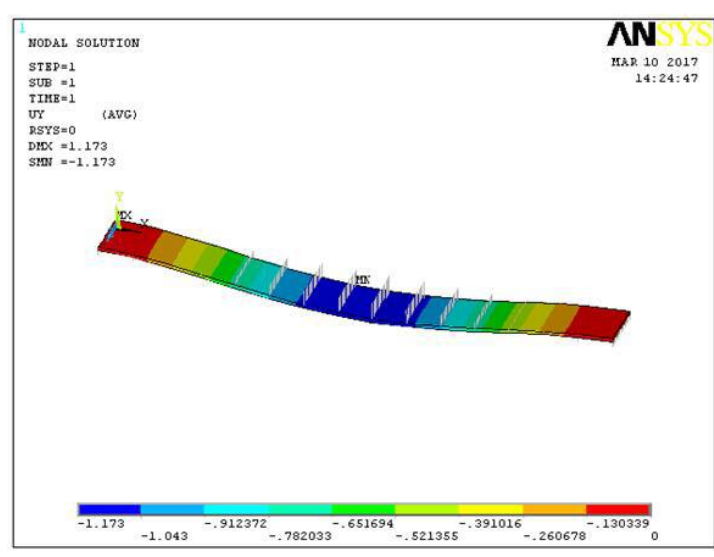

(a) Clamped beam thickness for $3 \mu \mathrm{m}$

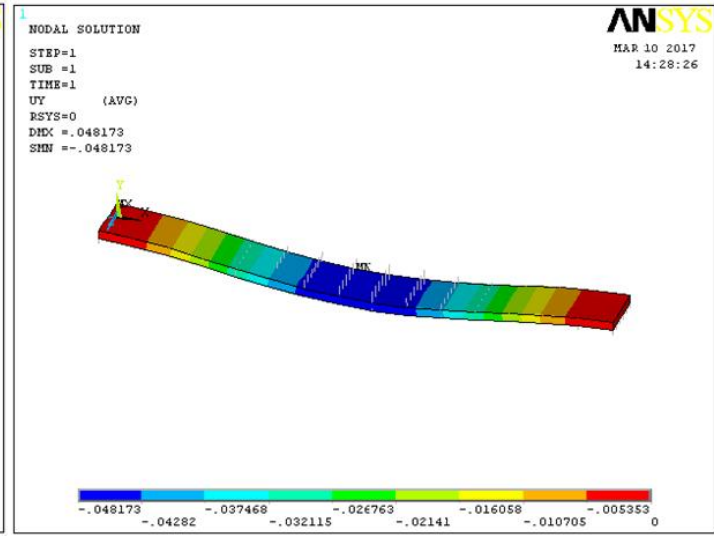

(b) Clamped beam thickness for $5 \mu m$
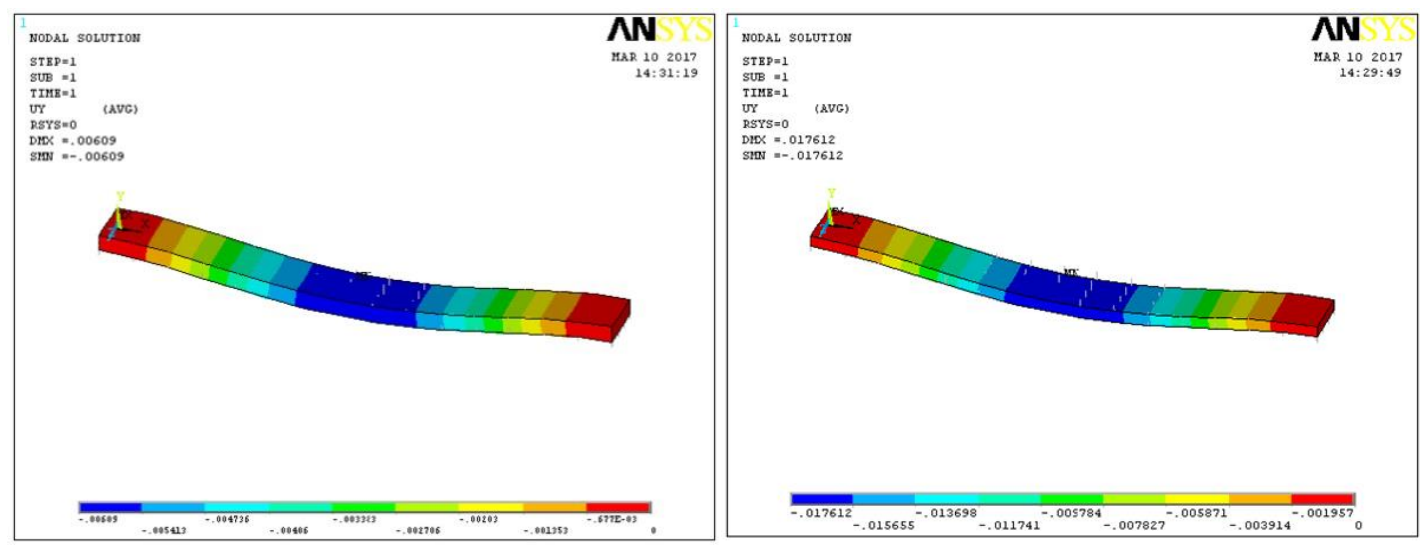

(c) Clamped beam thickness for $7 \mu m$

(d) Clamped beam thickness for $10 \mu \mathrm{m}$

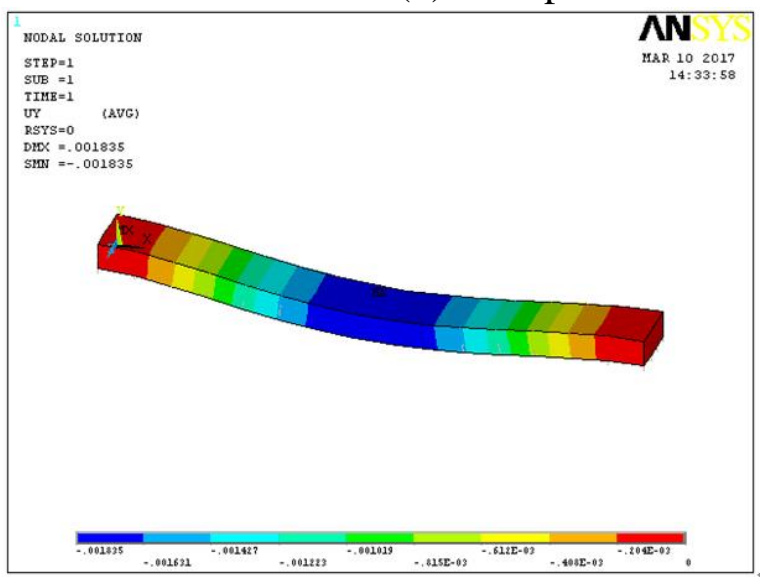

(e) Clamped beam thickness for $15 \mu \mathrm{m}$

Fig. 2 Ends clamped micro beam deformation figure in different thickness

Deformation analysis are carried out with ANSYS to establish the corresponding model is shown in figure 2, the fitting on the data obtained, obtained in the case of other parameters are fixed, the thickness of the micro beam clamped on both ends and the deflection deformation diagrams (see figure 3). Got by fitting curve can see $y \infty t^{-3}$, the maximum deformation of cantilever along with the increase of thickness of micro beam clamped at both ends and decrease rapidly. From the analysis of the five models can be concluded that under the same voltage, the deflection of $t=2 \mu m$ about $t=7 \mu \mathrm{m} 100$ times, 1000 times that of $t=15 \mu \mathrm{m}$. Describe the bending stiffness of the I EI is far less than other models. 


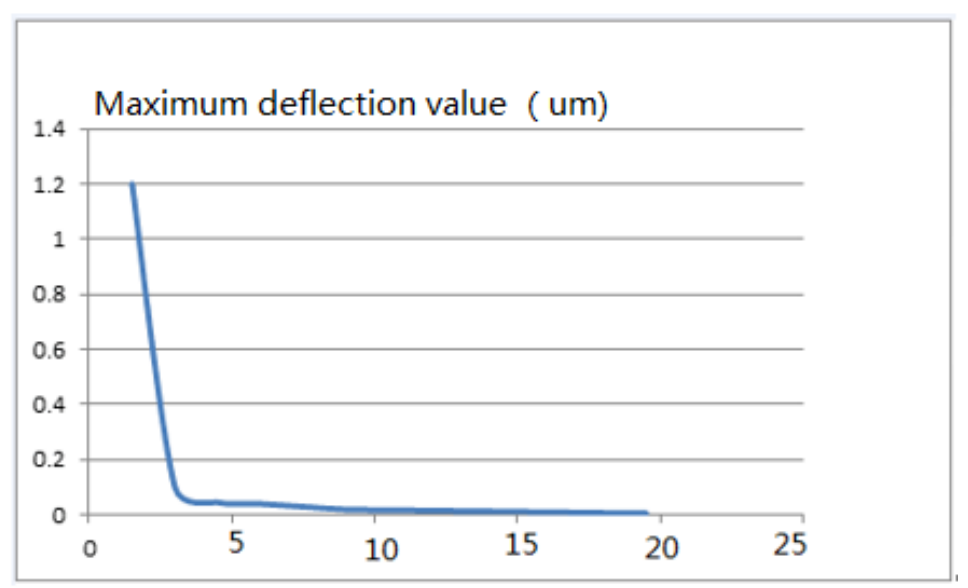

The thickness of clamped beam $\mathrm{h}(\mu \mathrm{m})$

Fig.3 Ends clamped cantilever thickness and maximum deflection of the relationship

When the beam structure size reaches the order of microns, the micro-beam will show different characteristics from the macroscopic field. Wang [4] and others, according to the strain gradient theory, analyze the micro-beam bending stiffness and macroscopic field of the different characteristics, the expression is: $I=E H^{3} /\left(12-12 v^{2}\right)+2 G l^{2} H, E H^{3} /\left(12-12 v^{2}\right)$ is the expression of stiffness under classical elasticity theory, and the other $2 G l^{2} H$ at the right is the contribution of gradient effect to bending stiffness. It can be seen that the bending stiffness of the micro-beam increases with the increase of the micro-beam thickness $\mathrm{H}$ when the characteristic length $l$ of the material remains constant. For the same voltage load, the micro-cantilever beams with different thickness and the two ends The results show that the bending stiffness of the micro - beam increases with the increase of the thickness, which is consistent with the theoretical analysis of Wang et al.

\section{Conclusion}

In this paper, the Trans126 degenerate element in ANSYS is used to simulate the fixed beam at both ends driven by electrostatic force. Under the same voltage load, the micro-beams with different thickness are analyzed, and the deflection of the micro- The micro-beam thickness increases and increases. The results show that the flexural rigidity of the micro - beam increases with the increase of the thickness, which is in accordance with the existing literature research results.

\section{References}

[1] Jianzhong fu, Xuxiao hu. Micro system principle and technology [M]. Beijing: mechanical industry press, 2005:142-143.

[2] L.Sun, X.E.Liu, X.J.Zheng, Numeriealsimulation of magnetostricitve film-substrate miero eantilever. Chin.J .Appl. Mech, 2004, 21:41- 45.

[3] Engel U, Eckstein R.Microforming-form basic research to its realization[J].Journal of Materials processing Technology, 2002, 125(1):35-44.

[4] Wang W, Huang Y. A study of microbend test by strain gradient plasticity [J].Int, J ,of Platicity, 2003(19):365-382. 